\title{
Development and application of culturally appropriate decision aids for smoking cessation in Korea: a pragmatic clustered randomization
} crossover trial

\author{
This article was published in the following Dove Press journal: \\ Patient Preference and Adherence \\ 22 September 2016 \\ Number of times this article has been viewed
}

\author{
Ji Eun Lee' \\ Dong Wook Shin ${ }^{1-3}$ \\ Beomseok Suh' \\ Sohyun Chun ${ }^{4}$ \\ You-Seon $\mathrm{Nam}^{3}$ \\ Belong Chol,2 \\ 'Department of Family Medicine, \\ Health Promotion Center, \\ ${ }^{2}$ Laboratory of Health Promotion and \\ Health Behavior, Biomedical Research \\ Institute, Seoul National University \\ Hospital, JJW Lee Center for Global \\ Medicine, Seoul National University \\ College of Medicine, ${ }^{4}$ International \\ Health Services, Samsung Medical \\ Center, Sungkyunkwan University \\ College of Medicine, Seoul, \\ Republic of Korea
}

Introduction: In Asian countries, reluctance to seek pharmacological intervention is a major barrier for smoking cessation. Culturally appropriate decision aids are expected to help people in the decision making for the use of smoking cessation medication.

Objective: The aim of this study was to develop a culturally tailored decision aid for smoking cessation and evaluate its effect on the use of smoking cessation medication.

Patients and methods: A 7-minute video on smoking cessation information and options was developed. Physicians were randomized into intervention and control groups. The decision aid was provided to patients in the intervention group, and they watched it, while those in the control group were provided usual medical care for smoking cessation. The primary outcome was the proportion of smokers who were prescribed smoking cessation medication within 1 month after consultation. The secondary outcomes were abstinence rate and use of smoking cessation medication within 6 months. A logistic regression analysis was used to assess the effect of the decision aid on the outcomes.

Results: In total, 414 current smokers (intervention group: 195; control group: 219) were enrolled. The mean age of the participants was 48.2 years, and 381 subjects ( $92 \%$ ) were males. In total, $11.8 \%$ of the participants in the intervention group and $10.5 \%$ in the control group were prescribed smoking cessation medications within 1 month. The odds ratio was 1.02 (95\% CI: 0.40-2.63) after adjustment for baseline characteristics. Within 6 months, $17.4 \%$ of the participants in the intervention group and $15 \%$ in the control group were prescribed medication (adjusted odds ratio 1.12, 95\% CI: 0.59-2.13).

Conclusion: The culturally tailored smoking cessation decision aid developed in this study did not show a significant impact on the decision to use smoking cessation medication. Further research to develop more effective and more interactive interventions is expected.

Keywords: smoking, clustered randomized trial, decision aids, Korea

\section{Introduction}

While pharmacological intervention, especially drug prescription, is recommended as the most effective therapy for smoking cessation, ${ }^{1,2}$ only a few smokers take such medications. This reflects a serious underutilization of the pharmacological intervention, ${ }^{3}$ which, in the Asian population, is as equally effective as that in their Western counterparts. ${ }^{4}$ However, data from the Korean National Health and Nutrition Examination Survey show that only $0.7 \%$ of current or former smokers reported that they had been prescribed smoking cessation medications, ${ }^{3}$ which is far less than the rates reported from the US, the UK,
Correspondence: Dong Wook Shin Department of Family Medicine, Seoul National University Hospital, I0I Daehak-ro, Jongno-gu, Seoul, Republic of Korea

Tel +82 220720847

Fax +8227663276

Email dwshin.snuh@gmail.com 
and Switzerland. ${ }^{5-7}$ Although the rate of smokers is decreasing in Korea, ${ }^{8}$ in 2013 , it was still estimated to be $22.8 \%$ in the general population and $42.1 \%$ in men. ${ }^{9}$ It would be important to encourage pharmacological therapy for smoking cessation, in order to decrease the smoking rate in Korea.

Culture might affect the treatment-seeking behavior related to smoking cessation. For example, reluctance to use smoking cessation medication has been identified as the most important barrier to successful smoking cessation in the general Korean population. ${ }^{10}$ In some multiethnic research studies, Asians were more reluctant to receive medical care for addictive disorders. ${ }^{11-13}$ They reported the fear of stigma after receiving medical support for such mental diseases. Especially in Korea, smoking has been considered just a habit and not a disorder. ${ }^{8}$ In addition, Asians tend to regard Western medicine as too "strong" for them and express the fear of side effects of such medication. ${ }^{12,14,15}$ These cultural factors may have been barriers for seeking pharmacological therapy for smoking cessation in Korea. Understanding the Korean smoker's perspectives on the methods of smoking cessation might be a key to the development of effective smoking cessation intervention for this population.

Patient decision aids are tools that help people become involved in the decision making by providing information about the options and outcomes and by clarifying personal values. They promote shared decision making between the patient and practitioner, save the physician's time, and reduce practice variation. Regarding smoking cessation, patient decision aids have been developed to help the patients decide on whether to quit smoking or not (National Heath Service, UK) and on whether to use smoking cessation medication or not (Healthwire, USA). ${ }^{16}$ However, such decision aids have targeted Western populations alone, thus limiting the generalizability of their utility in populations from other cultures. To the best of best knowledge, there is no decision aid specifically tailored for Korean smokers.

The objective of this study was to develop a culturally tailored decision aid for smoking cessation and to evaluate its effect on deciding to use smoking cessation medication. We expected that a culturally tailored smoking cessation decision aid would increase the usage of smoking cessation medication and enhance the smoking quit rate.

\section{Patients and methods}

\section{Patients}

The survey was conducted in the outpatient clinic of the Department of Family Medicine and the Health Screening Center of Seoul National University Hospital in Seoul, Republic of Korea. The participants included the patients who visited the physicians from March 2013 to January 2015. The inclusion criteria were patients who were currently smoking, having smoked at least one puff in the past 7 days, and being $\geq 18$ years old. Patients who were currently enrolled in other substance abuse treatment programs, who were pregnant, and who were not able to communicate in Korean were excluded. This study was approved by the institutional review board (IRB) of Seoul National University Hospital (IRB No 1202-032-397; Figure 1).

\section{Instruments and variables \\ Development of decision aids}

We developed a 7-minute long animated video containing information and options about smoking cessation. The contents were developed through a rigorous process of literature review, ${ }^{11,12,14,15,17-23}$ qualitative interviews with Korean smokers, and expert opinions from health care professionals. From the reviewed literature, it was suggested that Koreans regard smoking as a habit, not an addiction. ${ }^{10,11}$ In addition, the literature and qualitative interviews with smokers and experts revealed that Koreans have exaggerated concerns about the side effects of medication. Thus, in developing the decision aid, we focused on explaining smoking as nicotine dependency that needs clinical treatment and provided correct information, including the side effects of smoking cessation medication. The video contained 1) the risks of continued smoking; 2 ) the low success rate $(3 \%-5 \%)$ of an unaided quit attempt; 3) nicotine addiction and the role of the dopaminergic system; 4) the roles and options of smoking cessation medication; and 5) the options such as nicotine replacement therapy, bupropion, and varenicline. When presenting the options, information on the basic mechanisms and reported efficacy, the potential side effects, and medication costs were also included. The video concluded with an emphasis on the efficacy of smoking cessation medication therapy and an introduction of our smoking cessation clinic. The animation was developed such that it could be streamed on a tablet computer (iPad) at the clinic (Figure 2).

\section{Baseline variables}

We developed a questionnaire to assess baseline information. The questionnaire included the Fagerstrom test for nicotine dependence (FTND) to measure the level of nicotine dependency ${ }^{24}$ and the stage of change for smoking (no intention, precontemplation, contemplation, and preparation). ${ }^{25}$ The Korean version of the FTND was validated with high reliability (Cronbach's alpha $=0.69$ ) and adequate validity $\left(r=0.56\right.$ with expiratory carbon monoxide level). ${ }^{26}$ Self-assessed health (on a 5-point Likert scale), the level of 


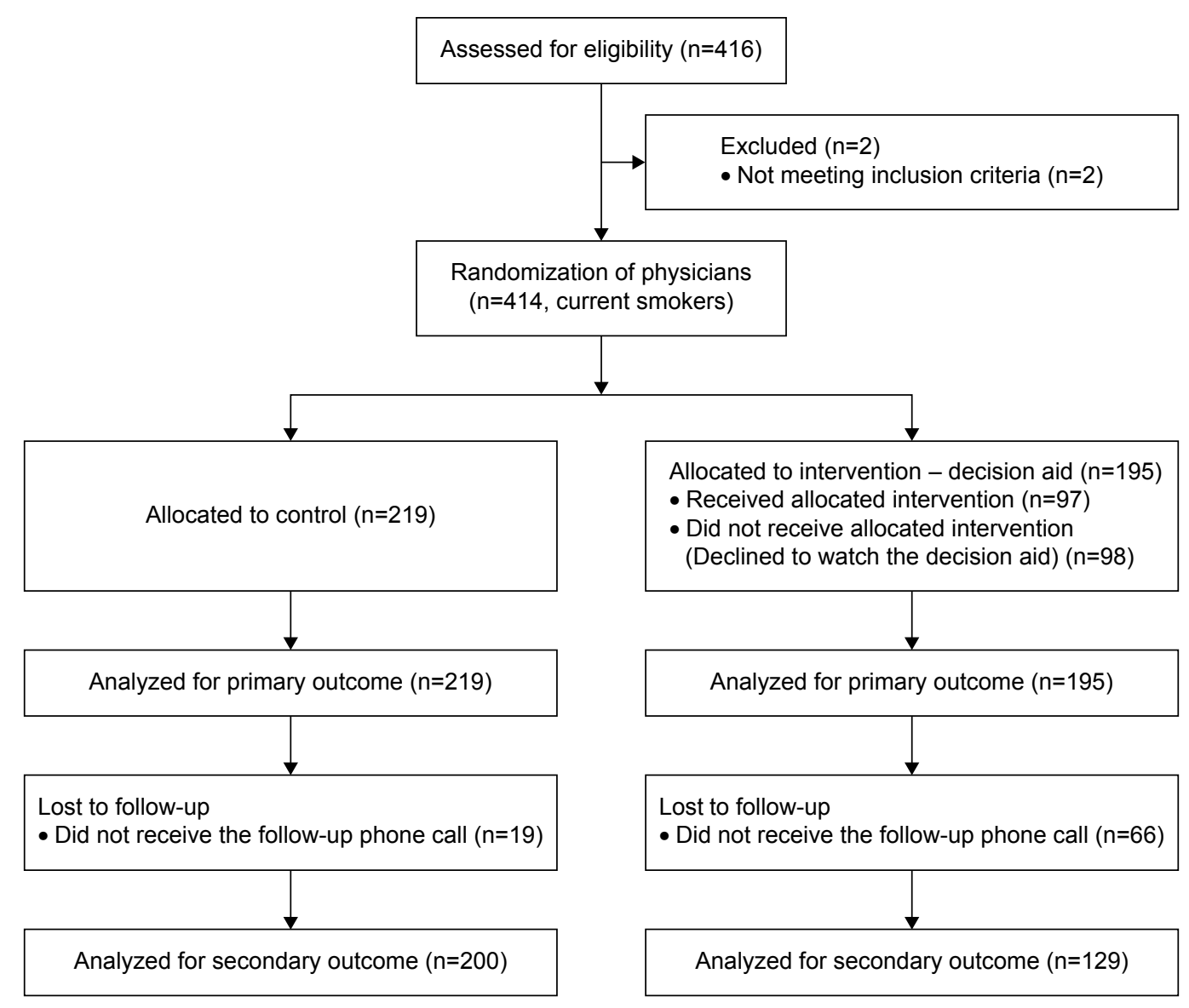

Primary outcome: proportion of smokers who were prescribed smoking-cessation medication within 1 month after the consultation

Secondary outcome: abstinence rate and use of smoking-cessation medication within 6 months after the consultation

Figure I Study scheme.

motivation, and self-efficacy for smoking cessation (10-point Likert scale, each) were enquired with each single-item rating scale, which are commonly used forms for studies of

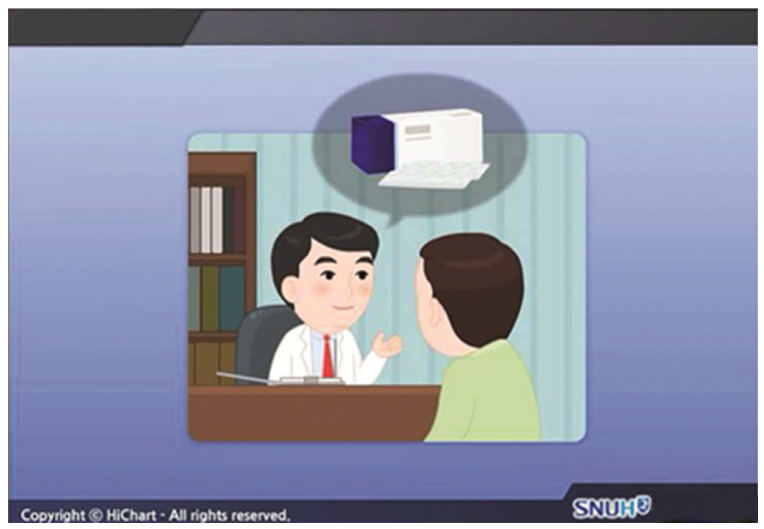

Figure 2 The decision aid developed and used in the present study.

Note: Used with permission of HealthBreeze (animation Link https:/lyoutu.bel El s0qts9HTI; short URL http://goo.gl/LNmCA2). health behavior change. ${ }^{27,28}$ In addition, we added questions on history of smoking (amount and years of smoking) and previous cessation attempts (number and mean values of attempts). The item on self-assessed health was used from the Korea National Health and Nutrition Examination Survey questionnaire. ${ }^{29,30}$ The questions on stage of change and motivation and self-efficacy for smoking cessation were developed by translation and back translation of the English items by the researchers of this study.

\section{Outcome variables}

The primary outcome of the study was the proportion of smokers who were prescribed smoking cessation medication within 1 month after the consultation. One month after the baseline visit, we reviewed the medical records of the participants to identify whether they were prescribed smoking cessation medications from Seoul National University Hospital, within the first month since the consultation. 
The secondary outcomes were the abstinence rate (point prevalence) and use of smoking cessation medication within 6 months. Six months after the initial consultation, we followed up the participants by telephone and reviewed their medical records from Seoul National University Hospital. We asked for their current smoking status and smoking amount if they were still smoking and the use of smoking cessation medication (regardless of whether the prescription was made in the same hospital or at other places). Those who quit smoking or reduced their smoking amount compared with the baseline amount were considered as reductions in smoking amount in the analyses.

\section{Procedure and data analysis Cluster randomization}

This study was designed as a clustered randomization trial. Physicians, not the patients, were randomized so that different practice styles for consultation among the physicians could be randomized. Only clinical fellows and third year residents of the Department of Family Medicine were included to minimize the physicians' cohort effect. In addition, since professors have higher clinical burden, it was impractical to include them in the study. The physicians were randomized by randomizing their consultation rooms. Each physician was assigned his/her own consultation room for each weekly consultation session for 1 year. Four consultation rooms in a row were assigned for the control and intervention groups in turns. The assignment was alternated each month to make a crossover design. In the even months, the even-numbered rooms were assigned to the intervention group, and in the odd months, the odd-numbered rooms were assigned to the intervention group. In this design, one physician was assigned to the intervention and control groups, alternatively, month by month. For example, if a physician was assigned Consultation Room 3, he/she was assigned to the control group in the even months and the intervention group in the odd months. Thus, the number of physicians in the two arms was same. The cluster size was 21 (Figure 1).

Patients' informed consent was waived by the IRB as our study intervention carried minimal risks for patients and the consent process itself could disproportionately influence the consent rate between the intervention and control groups. As patients in the intervention group had to watch a 7-minute video, it was expected that those who were less interested in smoking cessation might reject participation in the study. Especially, in intervention studies using educational programs, it has been recognized that the consent process itself can influence the volition to take the educational intervention, making the studies less scientific., ${ }^{31,32}$ Therefore, in many previous clustered randomization trials using educational materials for smoking cessation, the consent process was waived..$^{33-35}$ Thus, in the present study, physicians verbally explained the study procedures briefly when they provided consultations about smoking cessation and enrolled the patients who did not refuse to participate. This clinical trial was registered at ClinicalTrial.gov (identifier: NCT01566097).

\section{Sample size calculation}

We assumed that $\sim 10 \%$ of current smokers who visit a primary care clinic use smoking cessation medication (control) and that usage of smoking cessation medication would increase up to $25 \%$ with the intervention (decision aid). Assuming $80 \%$ power with 5\% significance level (twotailed), 100 subjects per group (200 subjects in total) would be necessary for a standard randomization trial. Adjusting for clustering effect by considering the mean cluster size of 21 and an intracluster correlation coefficient of $0.05,{ }^{36}$ the required sample was 200 subjects per group (400 subjects in total). ${ }^{37}$ The intracluster correlation coefficient values were 0.21 for the primary outcome variable (medication use in 1 month) and 0.10 for the secondary outcome variable (medication use in 6 months) in this study.

\section{Study procedure}

During enrollment, every participant filled a questionnaire. The routine assessment took only 1-2 minutes for most patients. After completing the questionnaire, the decision aid (7-minute video) was provided to the patients in the intervention group and they watched it. After that, physicians gave a brief consultation about smoking problems or prescribed medications if patients asked for it. If the patient did not show interest in smoking cessation, the physician only provided the routine medical care (on average, 5-15 minutes). If the patient was interested in smoking cessation, it took additional time for smoking cessation counseling and prescription (usually additional 5-10 minutes). The patients in the control group were not provided the decision aid and any proactive smoking cessation counseling or prescription. The usual medical care for the control group also took 5-15 minutes.

\section{Statistical analyses}

Baseline characteristics were summarized by descriptive statistics, mean, number, and rate. Each characteristic was compared between the control and intervention groups using a Student's $t$-test and chi-square test. To investigate the impact 
of the decision aid on the outcomes, univariate and multivariate logistic regression tests were used, with accounting for the clustering effect of nesting physicians (xtmelogit command of STATA for a multilevel analysis of binary outcome variables). The STATA software (Version 13.0; StataCorp LP, College Station, TX, USA) was used for all statistical analyses.

\section{Results}

\section{Participants' characteristics}

A total of 414 current smokers were enrolled, from which 195 were assigned to the intervention group and the remaining 219 patients to the control group. The mean age of the participants was 48.2 years and most of them were male $(92 \%)$. None of the characteristics was significantly different between the control and intervention groups (Table 1).

\section{Impact of the smoking cessation decision aid}

With reference to starting smoking cessation medication within 1 month, $11.8 \%$ of the participants in the intervention group and $10.5 \%$ in the control group were prescribed medication based on the medical record review. The odds ratio was 1.09 (95\% CI: $0.44-2.70)$ and it was $1.02(95 \%$ CI: 0.40-2.63) after adjusting for baseline characteristics. Within 6 months, $17.4 \%$ of the participants in the intervention group and $15 \%$ in the control group were prescribed medication based on self-report. The odds ratio was $1.12(95 \%$ CI: 0.59-2.13) in the multivariate model. Within the intervention group, 19.4\% quitted smoking within 6 months and 14\% did so in the control group. The odds ratio was $1.54(95 \%$ CI: $0.83-2.87)$ in the multivariate model. Almost the same proportion of participants reduced smoking amounts (45\% in the intervention group vs $45.5 \%$ in the control group). No other baseline characteristics were associated with the primary or secondary outcomes (Table 2).

\section{Discussion}

In the current study, we developed a culturally tailored smoking cessation decision aid and evaluated its effect on the decision to use smoking cessation medication. Contrary to our expectations, the decision aid did not show a significant impact on the decision to use smoking cessation medication. In addition, it did not affect quitting smoking and the amount reduction after 6 months.

Table I Baseline characteristics

\begin{tabular}{|c|c|c|c|c|c|c|c|}
\hline & \multicolumn{2}{|l|}{$\begin{array}{l}\text { Control } \\
(n=219)\end{array}$} & \multicolumn{2}{|c|}{$\begin{array}{l}\text { Intervention } \\
(n=195)\end{array}$} & \multicolumn{2}{|l|}{$\begin{array}{l}\text { Total } \\
(\mathrm{N}=4 \mid 4)\end{array}$} & \multirow[t]{2}{*}{ P-value } \\
\hline & $\bar{n}$ & $\%$ & $\bar{n}$ & $\%$ & $\bar{n}$ & $\%$ & \\
\hline Age (years), mean (SD) & $48.4(12.4)$ & & $48.0(13.3)$ & & $48.2(12.8)$ & & 0.763 \\
\hline \multicolumn{8}{|l|}{ Sex } \\
\hline Male & 197 & 90.0 & 184 & 94.4 & 381 & 92.0 & 0.099 \\
\hline Female & 22 & 10.1 & II & 5.6 & 33 & 8.0 & \\
\hline \multicolumn{8}{|l|}{ Smoking } \\
\hline Amount of smoking per day, mean (SD) & $17.6(10.0)$ & & $16.8(8.2)$ & & $17.2(9.2)$ & & 0.388 \\
\hline Years of smoking, mean (SD) & $25.6(11.3)$ & & 24.5 (II.9) & & $25.1(11.6)$ & & 0.315 \\
\hline Fagerstrom score, mean (SD) & $3.3(2.7)$ & & $3.5(2.7)$ & & $3.4(2.7)$ & & 0.408 \\
\hline \multicolumn{8}{|l|}{ Previous cessation attempt } \\
\hline Yes & 169 & 77.2 & $|4|$ & 72.3 & 310 & 74.9 & 0.255 \\
\hline Mean number of attempts (SD) & $3.3(3.6)$ & & $4.4(9.3)$ & & $3.8(6.8)$ & & 0.191 \\
\hline Unaided quit attempt & 121 & 55.3 & 110 & 56.4 & 231 & 55.8 & 0.813 \\
\hline Nicotine replacement therapy & 47 & 21.5 & 48 & 24.6 & 95 & 23.0 & 0.446 \\
\hline Prescription drug (bupropion, varenicline) & 16 & 7.3 & 13 & 6.7 & 29 & 7.0 & 0.799 \\
\hline Public health center (smoking cessation counseling) & 20 & 9.1 & 29 & 9.7 & 39 & 9.4 & 0.832 \\
\hline Quitline & 2 & 0.9 & 3 & 1.5 & 5 & 1.2 & 0.561 \\
\hline Herbal medicine/acupuncture & 12 & 5.5 & 9 & 4.6 & 21 & 5.1 & 0.689 \\
\hline No & 50 & 22.8 & 54 & 27.7 & 104 & 25.1 & \\
\hline \multicolumn{8}{|l|}{ Stages of change } \\
\hline Preparation stage & 80 & 38.7 & 77 & 41.2 & 157 & 39.9 & 0.271 \\
\hline Contemplation stage & 35 & 16.9 & 20 & 10.7 & 55 & 14.0 & \\
\hline Precontemplation stage & 79 & 38.2 & 73 & 39.0 & 152 & 38.6 & \\
\hline Do not want to stop smoking at all & 13 & 6.3 & 17 & 9.1 & 30 & 7.6 & \\
\hline Motivation, mean (SD) ${ }^{\mathrm{a}}$ & $6.8(2.5)$ & & $6.4(2.7)$ & & $6.6(2.6)$ & & 0.118 \\
\hline Self-efficacy, mean (SD) ${ }^{a}$ & $6.3(3.9)$ & & $6.2(2.5)$ & & $6.3(3.3)$ & & 0.795 \\
\hline
\end{tabular}

Note: aMotivation and self-efficacy for smoking cessation was assessed by a 10-point scale (I: not at all to 10 : very much). Abbreviation: SD, standard deviation. 
Table 2 Impact of the smoking cessation decision aid

\begin{tabular}{|c|c|c|c|c|c|c|}
\hline & \multicolumn{2}{|c|}{$\begin{array}{l}\text { Control } \\
(n=219)\end{array}$} & \multicolumn{2}{|c|}{$\begin{array}{l}\text { Intervention } \\
(\mathrm{n}=195)\end{array}$} & \multirow[t]{2}{*}{ OR $(95 \% \mathrm{CI})$} & \multirow[t]{2}{*}{ aOR $(95 \% \mathrm{Cl})^{2}$} \\
\hline & $\mathbf{n}$ & $\%$ & $\mathbf{n}$ & $\%$ & & \\
\hline \multicolumn{7}{|c|}{ Medication prescribed within I month (medical record) ${ }^{\mathrm{b}}$} \\
\hline Yes (prescribed) & 23 & 10.5 & 23 & 11.8 & $1.09(0.44-2.70)$ & $1.02(0.40-2.63)$ \\
\hline No & 196 & 89.5 & 172 & 88.2 & 1.00 & 1.00 \\
\hline \multicolumn{7}{|c|}{ Medication prescribed within 6 months (self-report) ${ }^{b}$} \\
\hline Yes & 30 & 15.0 & 24 & 17.4 & $1.26(0.68-2.35)$ & $1.12(0.59-2.13)$ \\
\hline No & 170 & 85.0 & 114 & 82.6 & 1.00 & 1.00 \\
\hline \multicolumn{7}{|c|}{ Smoking cessation after 6 months (self-report) } \\
\hline Yes & 28 & 14.0 & 25 & 19.4 & $1.48(0.82-2.67)$ & $1.54(0.83-2.87)$ \\
\hline No & 172 & 86.0 & 104 & 80.6 & 1.00 & 1.00 \\
\hline \multicolumn{7}{|c|}{ Smoking amount reduced after 6 months (self-report) } \\
\hline Yes & 91 & 45.5 & 58 & 45.0 & $0.98(0.62-1.56)$ & $0.97(0.59-1.57)$ \\
\hline No & 109 & 54.5 & 71 & 55.0 & 1.00 & 1.00 \\
\hline
\end{tabular}

Notes: ${ }^{2}$ Each analysis was adjusted for age, sex, Fagerstrom score, number of smoking cessation attempts, smoking cessation stage, and confidence for smoking cessation. 'Medication includes nicotine replacement therapy, bupropion, or varenicline. Abbreviations: OR, odds ratio; aOR, adjusted odds ratio.

In developing the decision aid, to identify the cultural barriers for use of smoking cessation medication unique to the Asian culture, especially in Korea, we reviewed previous studies ${ }^{11,12,14,15,17-23}$ and conducted qualitative interviews with smokers and smoking cessation experts. In one Korean study that investigated the physician's perception about current smoking cessation interventions and practice, patients' misunderstanding that "smoking is not an addiction but is just a habit" was recognized as the most common reason for smoking cessation treatment failure. ${ }^{10}$ Similarly, the shame in asking for help for an addictive disorder was identified as one of the most recognized cultural barriers in an Asian American population, leading to an underutilization of addiction treatment. ${ }^{11}$ Asians' general reluctance to use mental health services and their fear for the stigma of lacking willpower might be some hypothesized examples of culture-specific barriers to medication use. ${ }^{15,19-22}$ Especially in the Korean culture, which is strongly influenced by Confucianism, endurance and self-control are valued. ${ }^{17,19}$ This might affect helpseeking behavior for smoking cessation. In this context, we emphasized in the video aid that smoking is not just a habit but is nicotine dependency, which needs medical support.

Additionally, more universal barriers could be the lack of knowledge of possible treatment options and their efficacy and concerns about their side effects. In a study that analyzed the nationwide trends in using smoking cessation medication in Korea, there was an increasing trend for nicotine replacement therapy, which is an over-the-counter medication, but medication prescription did not show any increase. This confirms that such cultural barriers also exist in Korea. Meanwhile, education on smoking cessation was associated with smoking cessation use. ${ }^{3}$ Our qualitative interview with the smokers and physicians prior to the development of the decision aid also confirmed this barrier as the important one. Therefore, the decision aid was developed to address this issue specifically.

Furthermore, fear regarding the side effects of the medication and possible concerns regarding the cost of the medication might also affect the decision for seeking medication. In previous studies, Asians tended to regard Western medications as too strong for them and showed an immoderate fear regarding its side effects. Therefore, they would depend on Asian herbal medicine or waited for selfhealing. ${ }^{12,14,15}$ These issues were addressed within the decision aid by providing correct information on the side effects and cost of the medication.

Logically, we expected that our culturally tailored smoking cessation decision aid applied during routine primary care would increase the participants' knowledge about the efficacy of smoking cessation medications. We also anticipated people with positive attitudes toward smoking cessation medications would increase, encouraging people to discuss about smoking cessation medication with their physicians, and ultimately increase the usage of smoking cessation medication and enhance smoking quitting rate. However, the actual results were not consistent with our hypotheses.

The reasons for the absence of impact are not clear. There might be several reasons for the lack of efficacy. First, the decision aid was tailored for Korean cultural factors, but a personalized approach could not be achieved sufficiently. People have various reasons for continuing smoking and/or considering smoking cessation, and many smokers have ambivalent attitudes toward smoking cessation. Motivational interviewing, which is effective and is recommended for smoking cessation consultation, is a directive, client-centered counseling style for eliciting behavior change by helping 
clients to explore and resolve ambivalence. ${ }^{38}$ Our educational video material, which was provided to all the participants, could not adapt to such personal differences, and information on the efficacy, side effects, and costs of the smoking cessation medications are only few components of such a decisional balance for most smokers. Therefore, our standard video material might have not been sufficient to motivate the people to attempt quitting and to promote their decisions to use smoking cessation medication for their treatment. If we had routinely offered intensive smoking cessation consultation, such as motivational interviewing after seeing the educational video so that we could address their personal barriers, the decision aid could have had some synergistic effects. However, such a design would not allow us to evaluate the efficacy of our simple and scalable intervention per se.

Second, it could be possible that the information on the potential side effects and costs negatively affected the decision of the smokers to use smoking cessation treatment. From previous studies, the safety of smoking cessation medications was reported as a reason for their underuse. ${ }^{39}$ In the US, twothirds of smokers believed that "nicotine is as harmful as cigarettes are". ${ }^{39}$ In fact, after the FDA's communications about the possible safety concerns about varenicline, a decline in its use was observed. ${ }^{40}$ In addition, when the present study was conducted, the smoking cessation medications were not reimbursed in Korea, and the medication was not provided as a part of this study. The medication cost for varenicline or bupropion was -200-300 dollars for 12 weeks, which might be somewhat expensive for many smokers to pay for themselves. In Korea, before the medication was reimbursed in 2015, the high cost of smoking cessation medication was considered as one of the most important barriers for smoking cessation. We tried to provide not exaggerated, but correct information on smoking cessation medication. However, the information on the potential side effects and costs might have discouraged some smokers from deciding to start medication. Nonetheless, most patients desire to receive all the information concerning the possible adverse effects of any prescribed medication from the physicians. ${ }^{41}$ Physicians also need to explain both the pros and cons of a medication, as it is unfair to emphasize on the efficacy alone. Accordingly, the decision aid should address both sides adequately.

This study has some limitations. First, we did not investigate the smokers' attitudes toward smoking cessation treatment and medication before and after the study. This information might have been useful in the interpretation of our null results. Second, knowledge of treatment options was also not assessed before and after the study procedure, so knowledge improvement from watching the video aid was not assessed. Third, it was conducted in only one outpatient department of a tertiary referral hospital in Korea. Therefore, the findings were subject to selection bias and limited generalizability. Fourth, the smoking status was not verified by objective means such as urine nicotine concentration.

However, to our knowledge, this was the first study to adopt patient decision aids to promote smoking cessation medication by considering the important cultural barriers in Korea. Our study suggests that implementing a video decision aid developed to provide information on the smoking cessation medication, by considering the cultural context of reluctance in using medication for smoking cessation, in the context of routine primary care is not effective. While our results were not successful in increasing the use of smoking cessation medication or smoking cessation, we believe that our experience would be helpful for future researchers to develop more effective, more interactive, and personally tailored interventions.

\section{Acknowledgments}

The authors would like to thank Dr Hedoo Chung from HealthBreeze Company for producing the educational video for this study, and Giman Kim from Seoul National University Hospital for the administrative support provided during this study. This work was supported by a grant for investigator-initiated research from Pfizer (Pfizer Reference \#WS2033889). None of the sponsors had a role in any aspect of the present study, including design and conduct of study; collection, management, analysis, and interpretation of the data, and preparation, review, or approval of the manuscript. The study protocol and interim results of this study were presented at the 2015 Smoking Science Summit, held during May 19-21, 2015, at Peninsula Square, London, UK.

\section{Disclosure}

The authors report no conflicts of interest in this work.

\section{References}

1. West R, McNeill A, Raw M. Smoking cessation guidelines for health professionals: an update. Thorax. 2000;55(12):987-999.

2. Hung WT, Dunlop SM, Perez D, Cotter T. Use and perceived helpfulness of smoking cessation methods: results from a population survey of recent quitters. BMC Public Health. 2011;11(1):592.

3. Shin DW, Suh B, Chun S, et al. The prevalence of and factors associated with the use of smoking cessation medication in Korea: trend between 2005-2011. PLoS One. 2013;8(10):e74904.

4. Fagerström K, Nakamura M, Cho HJ, et al. Varenicline treatment for smoking cessation in Asian populations: a pooled analysis of placebocontrolled trials conducted in six Asian countries. Curr Med Res Opin. 2010;26(9):2165-2173.

5. Shiffman S, Brockwell SE, Pillitteri JL, Gitchell JG. Use of smokingcessation treatments in the United States. Am J Prev Med. 2008;34(2): 102-111.

6. Kotz D, Fidler J, West R. Factors associated with the use of aids to cessation in English smokers. Addiction. 2009;104(8):1403-1410. 
7. Etter JF, Perneger TV. Attitudes toward nicotine replacement therapy in smokers and ex-smokers in the general public. Clin Pharmacol Ther. 2001;69(3):175-183.

8. Lee HH. Present status and prospect of national policies on smoking cessation and prevention. Health and Welfare Policy Forum; 2007.

9. Ministry of Health and Welfare. Korea Health Statistics 2013: Korea National Health and Nutrition Examination Survey (KNHANES VI-1). Cheongju: Korea Centers for Disease Control and Prevention; 2014.

10. Kim CH, Song HR, Lee WS, Kim JY. Attitudes toward smoking cessation intervention and services among Korean physicians: a questionnaire survey. Korean J Fam Med. 2009;30(11):857-863.

11. Fong TW, Tsuang J. Asian-Americans, addictions, and barriers to treatment. Psychiatry (Edgmont). 2007;4(11):51-59.

12. Lin KM, Shen WW. Pharmacotherapy for Southeast Asian psychiatric patients. J Nerv Ment Dis. 1991;179(6):346-350.

13. Kim SS, Son H, Nam KA. Personal factors influencing Korean American men's smoking behavior: addiction, health, and age. Arch Psychiatr Nurs. 2005;19(1):35-41.

14. Ishisaka HA, Nguyen QT, Okimoto JT. The role of culture in the mental health treatment of Indochinese refugees. In: Owan TC, editor. Southeast Asian Mental Health: Treatment, Prevention, Services, Training and Research. Vol. 85. Washington, DC: US DHHS; 1985:54. DOCUMENT RESUME ED 266199 UD 024683.

15. Uba L. Cultural barriers to health care for Southeast Asian refugees. Public Health Rep. 1992;107(5):544.

16. Willemsen MC, Wiebing M, Van Emst A, Zeeman G. Helping smokers to decide on the use of efficacious smoking cessation methods: a randomized controlled trial of a decision aid. Addiction. 2006;101(3): 441-449.

17. Pang KYC. Symptoms of depression in elderly Korean immigrants: narration and the healing process. Cult Med Psychiatry. 1998;22(1): 93-122.

18. Hull SA, Cornwell J, Harvey C, Eldridge S, Bare PO. Prescribing rates for psychotropic medication amongst east London general practices: low rates where Asian populations are greatest. Fam Pract. 2001; 18(2):167-173.

19. Shin JK. Help-seeking behaviors by Korean immigrants for depression. Issues Ment Health Nurs. 2002;23(5):461-476.

20. Hussain FA, Cochrane R. Depression in South Asian women: Asian women's beliefs on causes and cures. Ment Health Relig Cult. 2002; 5(3):285-311.

21. Yeung A. How culture impacts on the treatment of mental illnesses among Asian-Americans. Psychiatric Times. 2004;21(1):34.

22. Donnelly PL. Mental health beliefs and help seeking behaviors of Korean American parents of adult children with schizophrenia. J Multicult Nurs Health. 2005;11(2):23.

23. Jones RS, Chow TW, Gatz M. Asian Americans and Alzheimer's disease: assimilation, culture, and beliefs. J Aging Stud. 2006;20(1):11-25.

24. Heatherton TF, Kozlowski LT, Frecker RC. The Fagerström test for nicotine dependence: a revision of the Fagerstrom Tolerance Questionnaire. Br J Addict. 1991;86(9):1119-1127.

25. DiClemente CC, Prochaska JO, Fairhurst SK, Velicer WF, Velasquez MM, Rossi JS. The process of smoking cessation: an analysis of precontemplation, contemplation, and preparation stages of change. J Consult Clin Psychol. 1991;59(2):295-304.
26. Ahn HK, Lee HJ, Jeong DS, Lee SY, Kim SW, Kang JH. Reliability and validity of Korean version of Fagerstrom Tolerance Questionnaire and Fagerstrom Test for Nicotine Dependence. J Korean Acad Fam Med. 2002;23(8):999-1008.

27. DiClemente CC, Nidecker M, Bellack AS. Motivation and the stages of change among individuals with severe mental illness and substance abuse disorders. J Subst Abuse Treat. 2008;34(1):25-35.

28. Hodgins D, Peden N, Makarchuk K. Self-efficacy in pathological gambling treatment outcome: development of a gambling abstinence self-efficacy scale (GASS). Int Gambl Stud. 2004;4(2):99-108.

29. Han M, Kim K, Park J, Kang M, Ryu S. Association between levels of physical activity and poor self-rated health in Korean adults: the Third Korea National Health and Nutrition Examination Survey (KNHANES), 2005. Public Health. 2009;123(10):665-669.

30. Kim M, Chung W, Lim S, et al. Socioeconomic inequity in self-rated health status and contribution of health behavioral factors in Korea. J Prev Med Public Health. 2010;43(1):50-61.

31. Puffer S, Torgerson D, Watson J. Evidence for risk of bias in cluster randomised trials: review of recent trials published in three general medical journals. BMJ. 2003;327(7418):785-789.

32. Eldridge SM, Ashby D, Feder GS. Informed patient consent to participation in cluster randomized trials: an empirical exploration of trials in primary care. Clin Trials. 2005;2(2):91-98.

33. Moore L, Campbell R, Whelan A, et al. Self help smoking cessation in pregnancy: cluster randomised controlled trial. BMJ. 2002; 325(7377): 1383 .

34. Lawrence T, Aveyard P, Cheng K, Griffin C, Johnson C, Croghan E. Does stage-based smoking cessation advice in pregnancy result in long-term quitters? 18-month postpartum follow-up of a randomized controlled trial. Addiction. 2005;100(1):107-116.

35. Aveyard P, Cheng K, Almond J, et al. Cluster randomised controlled trial of expert system based on the transtheoretical ("stages of change") model for smoking prevention and cessation in schools. $B M J$. 1999;319(7215):948-953.

36. Campbell MK, Mollison J, Steen N, Grimshaw JM, Eccles M. Analysis of cluster randomized trials in primary care: a practical approach. Fam Pract. 2000;17(2):192-196.

37. Sandra Eldridge [webpage on the Internet]. Sample Size in Cluster Randomised Trials. Available from: https://www.newton.ac.uk/ files/seminar/20110815152516051-152821.pdf. Accessed January 8, 2013.

38. Rollnick S, Miller WR. What is motivational interviewing? Behav Cogn Psychother. 1995;23(04):325-334.

39. Shiffman S, Ferguson SG, Rohay J, Gitchell JG. Perceived safety and efficacy of nicotine replacement therapies among US smokers and ex-smokers: relationship with use and compliance. Addiction. 2008; 103(8):1371-1378.

40. Kasza KA, Cummings KM, Carpenter MJ, Cornelius ME, Hyland AJ, Fong GT. Use of stop-smoking medications in the United States before and after the introduction of varenicline. Addiction. 2015;110(2): 346-355.

41. Ziegler DK, Mosier MC, Buenaver M, Okuyemi K. How much information about adverse effects of medication do patients want from physicians? Arch Intern Med. 2001;161(5):706-713.
Patient Preference and Adherence

\section{Publish your work in this journal}

Patient Preference and Adherence is an international, peer-reviewed, open access journal that focuses on the growing importance of patient preference and adherence throughout the therapeutic continuum. Patient satisfaction, acceptability, quality of life, compliance, persistence and their role in developing new therapeutic modalities and compounds to optimize

\section{Dovepress}

clinical outcomes for existing disease states are major areas of interest for the journal. This journal has been accepted for indexing on PubMed Central. The manuscript management system is completely online and includes a very quick and fair peer-review system, which is all easy to use. Visit http://www dovepress.com/testimonials.php to read real quotes from published authors. 